\title{
Intracranial hypertension after Chiari decompression resolving after removal of a levonorgestrel-releasing intrauterine device: case report
}

\author{
Georgios A. Maragkos, MD, Rouzbeh Motiei-Langroudi, MD, Aristotelis S. Filippidis, MD, PhD, \\ and Efstathios Papavassiliou, MD
}

Division of Neurosurgery, Beth Israel Deaconess Medical Center, Harvard Medical School, Boston, Massachusetts

Levonorgestrel-releasing intrauterine devices (LIUDs) are thought to release this progestin locally in the uterus to limit side effects. Authors here present a case of treatment-refractory hydrocephalus and pseudomeningocele (PMC), both of which fully resolved after LIUD removal.

A 35-year-old woman with an implanted LIUD developed symptomatic PMC and hydrocephalus after suboccipital craniectomy for Chiari malformation type I. Over the next 8 months, she underwent ventriculoperitoneal shunt placement and two attempts at needle decompression of the fluid collection, which did not relieve her symptoms or the PMC, except for a few days at a time. Subsequently, she had her LIUD removed. Three weeks after removal of the LIUD, her symptoms as well as the fluid collection resolved completely without any further intervention. Thus, the increased intracranial pressure and associated persistence of the PMC may be partially attributed to the LIUD.

This case indicates that a persistent problem (PMC and intracranial hypertension) that may be associated with the LIUD rapidly resolves after its removal. Implication of LIUDs as the cause of intracranial hypertension is still a matter of controversy. Further studies are needed to evaluate any potential causal relationship between LIUDs and intracranial hypertension, and physicians are advised to consider this scenario in their differential diagnosis.

https://thejns.org/doi/abs/10.3171/2018.5.JNS18315

KEYWORDS pseudomeningocele; levonorgestrel-releasing intrauterine device; Chiari decompression; hydrocephalus

$\mathrm{P}$ ROGESTINS are frequently used in various formulations for female contraception. The association of progestins with increased intracranial pressure (ICP) in the form of idiopathic intracranial hypertension (IIH), also known as "pseudotumor cerebri", has been demonstrated. ${ }^{3,5}$ However, there has never been proof of an association between progestins and hydrocephalus. Levonorgestrel-releasing intrauterine devices (LIUDs) were originally thought to release progestins only locally in the uterus, decreasing systemic absorption and related side effects, ${ }^{8}$ but it has been shown that these devices may result in similar hormonal blood levels as systemic progestin formulations. ${ }^{4,10}$ Moreover, LIUDs have also been associated with the development of IIH..${ }^{3,5}$ Here we present the case of a 35-year-old woman with an implanted LIUD who developed a symptomatic pseudomeningocele (PMC) following Chiari decompression, which was refractory to multiple treatment modalities for 8 months. Three weeks after removal of the LIUD, her symptoms as well as the fluid collection had completely resolved. Thus, the increased ICP may be partially attributed to the LIUD. To our knowledge, there is no report of treatment-refractory intracranial hypertension and PMC resolving after removal of an LIUD.

\section{Case Report}

History and Examination

A 35-year-old, right-handed woman was referred to the neurosurgery clinic with 1 month of progressive numbness

ABBREVIATIONS ICP = intracranial pressure; IIH = idiopathic intracranial hypertension; $\mathrm{LIUD}=$ levonorgestrel-releasing intrauterine device; PMC = pseudomeningocele; VPS = ventriculoperitoneal shunt.

SUBMITTED February 2, 2018. ACCEPTED May 29, 2018.

INCLUDE WHEN CITING Published online October 19, 2018; DOI: 10.3171/2018.5.JNS18315. 

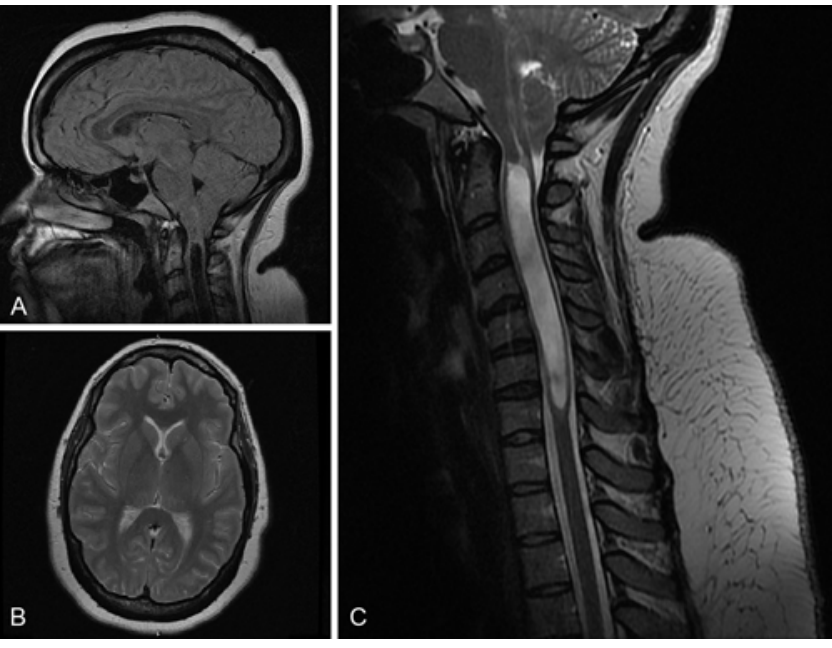

FIG. 1. Preoperative imaging. A: Sagittal brain T1-weighted MR image showing herniation of the cerebellar tonsils about $1.7 \mathrm{~cm}$ below the foramen magnum and a large syrinx in the cervical spine. B: Axial brain T2-weighted MR image showing small lateral ventricles. C: Sagittal cervical spine T2-weighted MR image showing a large syrinx extending from $\mathrm{C} 1$ to $\mathrm{C} 7$.

and tingling of the left hand, left side of the chest and abdomen, and right side of the face. Pertinent medical history included obesity (body mass index $48.3 \mathrm{~kg} / \mathrm{m}^{2}$ ), hypertension, hyperlipidemia, 90 pack-years of smoking, and placement of an LIUD containing $52 \mathrm{~g}$ of levonorgestrel (Mirena, Bayer Schering Pharma) 6 years before presentation. Magnetic resonance imaging revealed significant cerebellar tonsillar herniation $17 \mathrm{~mm}$ below the foramen magnum and a cervical spine syringomyelia $9.1 \mathrm{~cm}$ long and $7 \mathrm{~mm}$ wide, extending to the $\mathrm{C} 7$ level as well as the small lateral ventricles (Fig. 1). The patient was diagnosed with Chiari malformation type I and underwent Chiari decompression without complications. The postoperative hospital course was uncomplicated, and the patient was discharged 3 days later, reporting no symptoms.

Over the next 2 months, she developed an occipital fluid collection, which progressively increased to $8 \mathrm{~cm}$ in diameter, along with the reappearance of the left upper extremity paresthesias, new-onset persistent headache, nausea, and vomiting. Computed tomography scanning of her head revealed hydrocephalus and a PMC (Fig. 2). Symptomatic treatment with antiemetics and reevaluation of her pain regimen failed to provide relief.

\section{Operation and Postoperative Course}

She then underwent right-sided programmable ventriculoperitoneal shunt (VPS) placement (Strata II, Medtronic PLC), with the shunt set at 1.0. Cerebrospinal fluid analysis via lumbar puncture 1 day prior to the operation was consistent with possible chemical meningitis $(86 \mathrm{mg} /$ dl protein, 123 leukocytes/dl, no growth on culture). Six weeks later, she continued to experience constant moderate-intensity headaches, as well as new-onset ataxia. Computed tomography revealed appropriate VPS placement, interval improvement of the hydrocephalus, syringomyelia decreased to $5 \times 6 \mathrm{~mm}$, effacement of the extraaxial spaces supra- and infratentorially, and no change in PMC size (Fig. 3). The shunt was reprogrammed to the lowest value of 0.5 .

Two months later, there was still no symptomatic improvement. Office-based needle aspiration of the fluid collection yielded $130 \mathrm{ml}$ of clear, yellow fluid, and the patient was scheduled for follow-up. There was no improvement in her headaches and nausea, and 2 days later she stated over the phone that the fluid had reaccummulated. Needle aspiration of the collection was repeated 2 months later, yielding $180 \mathrm{ml}$ of straw-colored fluid. Again, the fluid reaccummulated in 2 days with no symptomatic improvement. Fluid cultures were negative in both cases. She was scheduled for follow-up in 2 months, and reoperation for duraplasty revision was considered.

Fifteen days later, the patient had her LIUD removed by her primary care provider. Although no further aspirations or any other management was attempted, her symptoms gradually improved over the next 3 weeks. Six weeks after LIUD removal, she had complete resolution of her occipital CSF collection; significant improvement in her headaches, ataxia, and paresthesias; and resolution of her nausea and vomiting. The shunt was at 0.5 at the time of
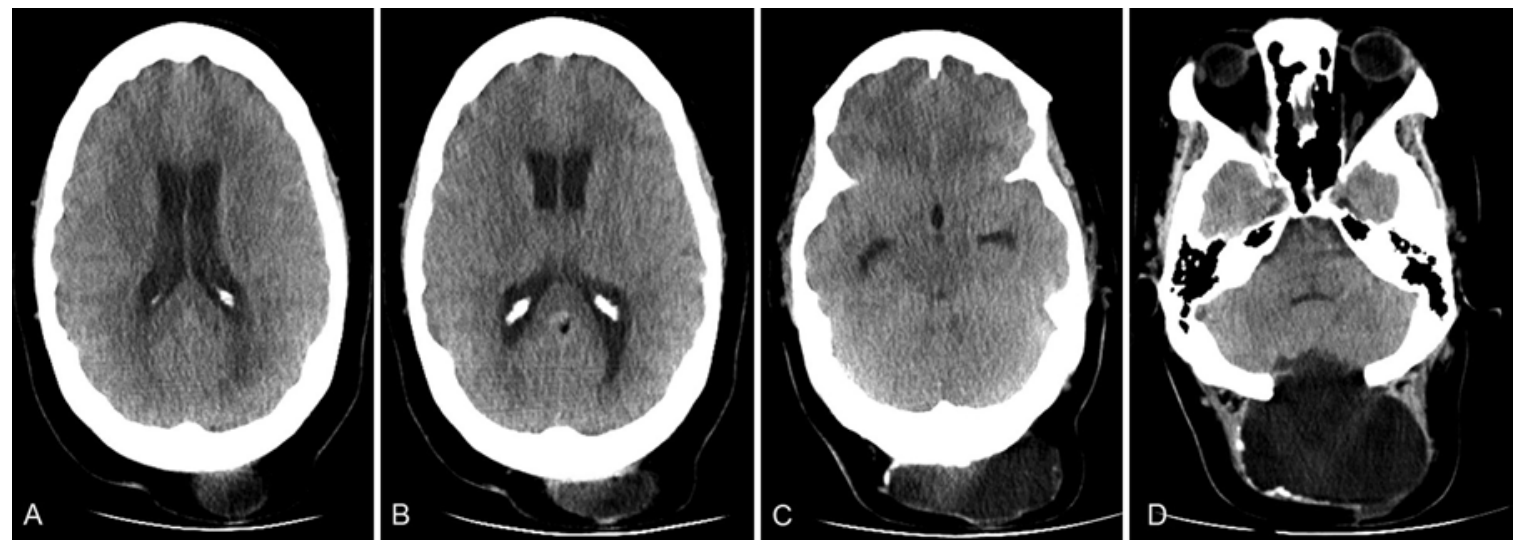

FIG. 2. Postoperative follow-up axial skull CT images obtained at various levels (A-D), showing bilateral enlargement of the lateral ventricles, effacement of the sulci, and a significant suboccipital CSF collection consistent with PMC. 

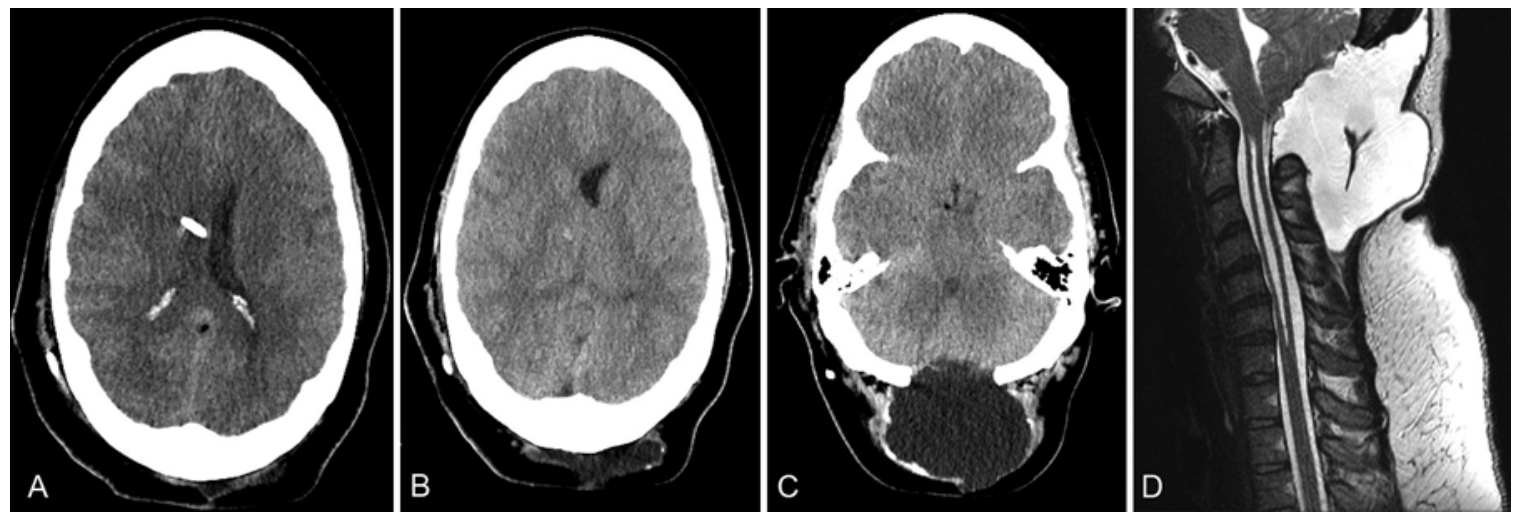

FIG. 3. Follow-up axial CT scans (A-C) obtained after VPS placement, showing resolution of hydrocephalus on the right, VPS placement in the right lateral ventricle, effacement of the sulci, and persistence of the suboccipital PMC. Sagittal T2-weighted MR image (D) showing improvement of the cervical syringomyelia and persistence of the PMC.

IUD removal and was set to 1.0 after resolution of her symptoms.

\section{Discussion}

The most common sequelae after decompressive surgery for Chiari malformation type I are PMC and persistent CSF leakage through the dural graft, possibly exacerbated by hydrocephalus and raised ICP. 6 "Pseudomeningocele" refers to the formation of a collection of $\mathrm{CSF}$ in the occipital-cervical area, which is directly related to the durotomy. Most cases of postoperative PMC will resolve without treatment if they are asymptomatic, but symptomatic and persistent cases may require prompt management with fluid aspiration or duraplasty revision. ${ }^{6}$

Here we describe a patient with treatment-refractory PMC and high ICP, resolving after removal of her LIUD. The patient underwent occipital decompression for the Chiari malformation and subsequently developed hydrocephalus and a PMC through the cranial defect. However, the PMC did not resolve after multiple treatment modalities, including placement of a VPS set at low pressure and repeated tapping of the fluid collection. Notably, the Chiari decompression was successful, as evidenced by the decrease in syringomyelia size. Eight months after the original operation, the patient had her LIUD removed, which resulted in an abrupt and complete resolution of her symptoms in 3 weeks without any additional intervention.

Progestins have been associated with the development of IIH with an unknown mechanism. ${ }^{1,3,4,7}$ In 1995 Alder et al. reported on the risk of IIH in women implanted with subdermal levonorgestrel and found a total of 2 cases of IIH at their institution and an additional 56 cases in various databases. ${ }^{1}$ Additionally, Chan described a case of symptomatic IIH 2 months after initiating depot medroxyprogesterone. ${ }^{2}$ The symptoms and high ICP resolved completely 4 months after discontinuation of the medication.

Levonorgestrel-releasing IUD implants were previously thought to release most of the drug topically, with lower amounts systemically absorbed, reducing the risk of side effects. This notion has been the subject of debate. Levonorgestrel binds to sex hormone-binding protein (SHBP), the concentrations of which can vary among women, causing variable systemic concentrations. ${ }^{3}$ Furthermore, serum levels of levonorgestrel have been shown to be similar between LIUDs and oral levonorgestrel formulations. ${ }^{4}$ Idiopathic intracranial hypertension has also been shown to occur as a side effect after LIUD placement, ${ }^{3}$ implying that LIUDs may, in fact, account for side effects similar to those of systemically administered progestin formulations.

The case illustrated here is the first report of a possible association of LIUD with hydrocephalus and increased ICP. This patient had an LIUD placed 6 years before presentation and would possibly have a stable systemic level of progestins by the time of presentation. Patients undergoing Chiari decompression should be screened for hydrocephalus, IIH, and increased ICP before surgery because these cases are prone to complications including PMC and persistent CSF leakage. ${ }^{6}$ Our patient may have had subclinical baseline intracranial hypertension because of the LIUD, as supported by her preoperative MRI and body habitus. This then became clinically significant (overt hydrocephalus and PMC) after developing chemical meningitis following Chiari decompression.

The fact that the PMC was refractory to other forms of treatment but did involute after LIUD removal may implicate the LIUD in the pathophysiology of this patient's condition. Progestins are known to cause increased ICP in some patients, and whether LIUDs release enough progestins systemically is still a matter of debate. If that is the case, it is plausible that the refractoriness of this patient's complication and the inability of the shunt at its lowest setting to resolve her symptoms may, in part, be attributed to the increased ICP from her LIUD. This may account for the resolution of her symptoms 3 weeks after removal of the LIUD. Furthermore, the cause-effect relationship between hydrocephalus and Chiari malformation is still a matter of debate. ${ }^{6,9}$ However, the possibility of the LIUD causing subclinical hydrocephalus, which led to the Chiari malformation, could also be entertained.

Our study entails the intrinsic limitations of case reports, namely the lack of statistical sampling and a control group for comparison. While this case report cannot conclusively prove an etiological relationship, all necessary 
measures to treat hydrocephalus and PMC were taken, and the last thing that was changed was removing the LIUD; therefore, the possibility of a causal relationship should be reported.

\section{Conclusions}

This case report is the first to demonstrate a plausible cause for refractory postcraniectomy PMC associated with increased ICP. While the implication of LIUDs as the cause of IIH and increased ICP is still a matter of debate, clinicians should be alerted to that possibility. This case report cannot prove an association, but we show that removal of the LIUD led to rapid resolution of the patient's symptoms and refractory PMC collection. Further studies are needed to evaluate the causal relationship between LIUDs and increased ICP, and physicians are advised to consider this scenario in their differential diagnosis.

\section{References}

1. Alder JB, Fraunfelder FT, Edwards R: Levonorgestrel implants and intracranial hypertension. N Engl J Med 332:1720-1721, 1995

2. Chan JW: Idiopathic intracranial hypertension associated with depot medroxyprogesterone. Eye (Lond) 20:1396-1397, 2006

3. Etminan M, Luo H, Gustafson P: Risk of intracranial hypertension with intrauterine levonorgestrel. Ther Adv Drug Saf 6:110-113, 2015

4. Ewies AA: Levonorgestrel-releasing intrauterine systemthe discontinuing story. Gynecol Endocrinol 25:668-673, 2009

5. Friedman D: Risk of intracranial hypertension with intrauterine levonorgestrel. Ther Adv Drug Saf 7:23-24, 2016

6. Heiss J, Oldfield E: Treatment of the adult Chiari I malformation, in Tubbs RS, Oakes WJ (eds): The Chiari Malformations. New York: Springer, 2013, Vol 1, pp 291-306

7. Ireland B, Corbett JJ, Wallace RB: The search for causes of idiopathic intracranial hypertension. A preliminary casecontrol study. Arch Neurol 47:315-320, 1990

8. Kailasam C, Cahill D: Review of the safety, efficacy and patient acceptability of the levonorgestrel-releasing intrauterine system. Patient Prefer Adherence 2:293-302, 2008

9. Shoja MM, Johal J, Oakes WJ, Tubbs RS: Embryology and pathophysiology of the Chiari I and II malformations: a comprehensive review. Clin Anat 31:202-215, 2018

10. Xiao B, Wu SC, Chong J, Zeng T, Han LH, Luukkainen T: Therapeutic effects of the levonorgestrel-releasing intrauterine system in the treatment of idiopathic menorrhagia. Fertil Steril 79:963-969, 2003

\section{Disclosures}

The authors report no conflict of interest concerning the materials or methods used in this study or the findings specified in this paper.

\section{Author Contributions}

Conception and design: Papavassiliou, Maragkos, Motiei-Langroudi. Acquisition of data: Papavassiliou, Maragkos, MotieiLangroudi. Analysis and interpretation of data: Maragkos, Motiei-Langroudi, Filippidis. Drafting the article: Maragkos, Motiei-Langroudi, Filippidis. Critically revising the article: all authors. Reviewed submitted version of manuscript: Papavassiliou. Approved the final version of the manuscript on behalf of all authors: Papavassiliou. Study supervision: Papavassiliou.

\section{Supplemental Information}

Previous Presentations

Portions of this work were presented in poster form at the American Association of Neurological Surgeons Annual Scientific Meeting held in New Orleans, LA, in 2018.

\section{Correspondence}

Efstathios Papavassiliou: Beth Israel Deaconess Medical Center, Harvard Medical School, Boston, MA. epapavas@bidmc.harvard. edu. 